\section{Programa Saúde da Família: acerca da adequação do uso do seu indicador de cobertura}

\author{
The Family Health Program in Brazil and the \\ adequacy of its coverage indicator
}

\author{
1 Instituto de Saúde Coletiva, \\ Universidade Federal da \\ Bahia, Salvador, Brasil. \\ Correspondência \\ R. Aquino \\ Instituto de Saúde Coletiva \\ Universidade Federal da \\ Bahia. \\ Rua Basílio da Gama s/n, \\ Salvador, $B A$ \\ 40110-040, Brasil. \\ aquino@ufba.br
}

\begin{abstract}
This study aimed to evaluate the adequacy of the Family Health Program's (FHP) coverage indicator, calculated by using the estimated number of people served by the FHP team, obtained from a national standard. The study included all the Brazilian counties (municipalities) that adopted the FHP strategy from 1998 to 2006. The analytical methods were Pearson's correlation, Lin's concordance correlation coefficient, and the Deming regression model. The results showed that at the beginning of the observation period, the coverage indicator did not express the size of the program's population of beneficiaries, when comparing the enrolled population, the number of families under follow-up, and the number of live births recorded. Gradually, and especially beginning in 2000, the indicator began to represent a good estimate of FHP coverage, although the trend differed according to the size of the municipalities (it was more evident among the larger municipalities). The study emphasizes the importance of defining coverage of actions and services in order to orient health program implementation.
\end{abstract}

Family Health Program; Health Services Coverage; Program Evaluation
Rosana Aquino 1

Mauricio L. Barreto 1

\section{Introdução}

Nas avaliações de efetividade de programas e serviços de saúde, a intervenção é geralmente definida através de um indicador de cobertura. Ao mesmo tempo em que estabelece a própria existência das ações e serviços, a quantificação da cobertura expressa a magnitude da implantação das ações em um dado território ou população, constituindo-se, enquanto variável independente principal, em um dos componentes fundamentais do modelo conceitual a ser testado empiricamente.

Assim, a definição de indicadores de cobertura válidos e precisos deveria representar uma etapa fundamental do monitoramento e avaliação de saúde e pré-condição para o desenvolvimento dos estudos de efetividade. Dada a aparente simplicidade da formulação e obtenção de indicadores de cobertura, as questões teórico-conceituais, metodológicas e operacionais envolvidas na construção de boas estimativas do parâmetro não raro são negligenciadas por avaliadores e gestores de saúde.

Há diferentes conceitos de cobertura, dependendo da perspectiva adotada, se econômica, programática, demográfica ou geográfica. $\mathrm{O}$ conceito mais utilizado toma por base o universo de usuários, distinguindo a cobertura em: legal, corresponde à população que tem direito aos serviços; potencial, à população adstrita às unidades de saúde; e real, à população que faz uso 
dos serviços. Outros enfoques levam em conta a disponibilidade de recursos, o tipo ou quantitativo de serviços desejáveis, a relação entre população realmente atendida e população que deveria ser atendida e a satisfação das necessidades de saúde da população ${ }^{1}$.

Além disso, é crucial determinar a população-alvo que deveria se beneficiar das ações e serviços de saúde ofertados no processo de implantação e implementação de um programa 2,3. Gerenciar um programa efetivamente requer garantir que a população usuária do projeto corresponda à população-alvo e que a cobertura seja mantida em níveis aceitáveis, o que envolve, inclusive, atenção especial quanto à localização geográfica das unidades de saúde.

No Brasil, o Programa Saúde da Família (PSF) tem adotado uma estimativa para o cálculo da população coberta a partir de um parâmetro nacional único: o número médio estimado de pessoas acompanhadas por uma equipe de saúde da família (3.450 pessoas). Este indicador vem sendo utilizado nas avaliações institucionais, em pesquisas avaliativas e pelos técnicos das esferas de gestão do Sistema Único de Saúde (SUS), inclusive para definir o volume de recursos referentes aos incentivos federais e estaduais do PSF, pelas Secretarias Estaduais de Saúde e Ministério da Saúde. Mesmo as Secretarias Municipais de Saúde, que dispõem do número de pessoas ou famílias cadastradas ou acompanhadas por equipe, através do Sistema de Informação da Atenção Básica (SIAB), habitualmente, não corrigem a estimativa 4 .

Uma vez que, em cada município, o parâmetro estimado pode não corresponder à população realmente atendida e beneficiária das ações e serviços, esta opção poderia acarretar problemas de validade conceitual e operacional que devem ser mensurados. Este estudo tem como objetivo investigar a adequação do uso deste indicador de cobertura do PSF (número estimado de pessoas acompanhadas por equipe dividido pela população total do município), analisando a sua correlação e concordância com outros indicadores relacionados ao funcionamento do programa nos municípios brasileiros.

\section{Metodologia}

O estudo abrangeu todos os municípios brasileiros com o PSF em funcionamento, por pelo menos um ano, no período de 1998 a 2006. Para cada ano foram obtidas informações sobre a cobertura do PSF e indicadores de cadastramento e registro da população assistida pelo programa - percentual da população cadastrada, percen- tual de famílias acompanhadas e percentual de nascidos vivos registrados. Foram realizadas análises de correlação e concordância entre o indicador de cobertura do PSF e os demais indicadores para cada ano do período estudado, o que permitiu verificar a tendência das relações entre estes indicadores no tempo.

\section{Definição das variáveis e fontes de dados}

A cobertura do PSF foi obtida através do produto do número de equipes de saúde da família implantadas no mês de dezembro de cada ano (ou no último mês do ano com informações do município) pelo número médio estimado de pessoas acompanhadas por equipe (3.450 pessoas, segundo padrão adotado pelo Departamento da Atenção Básica do Ministério da Saúde) dividido pela população total do município. As informações referentes ao período de 1998 a 2000 foram obtidas do Sistema de Captação de Dados para Pagamento (CAPSI, criado para o cálculo e pagamento de incentivos financeiros ao Programa de Agentes Comunitários - PACS e PSF em 1998 e desativado em 2000) e, a partir de 2001, do SIAB, fonte de informação que passou a ser adotada para esta finalidade pelo Ministério da Saúde.

O percentual da população cadastrada no SIAB foi obtido pela razão do número de pessoas cadastradas no SIAB em dezembro de cada ano pelo total da população do município no ano correspondente.

O percentual de famílias acompanhadas pelas equipes de saúde da família foi obtido pela razão do número de famílias acompanhadas (média anual das informações mensais fornecidas pelo município no SIAB), do número total de famílias do município, estimado pela divisão da população do município pela média de pessoas por família, conforme a distribuição regional segundo o Censo Demográfico 2000 (Instituto Brasileiro de Geografia e Estatística - IBGE; http://www.ibge. gov.br) (4,0 pessoas por família na Região Norte; 3,7 pessoas no Nordeste; 3,3 no Sudeste e no Sul; e 3,4 no Centro-Oeste).

O percentual de nascidos vivos informados no SIAB foi obtido pela razão do total de nascidos vivos informados pelo SIAB do total de nascidos vivos informados no Sistema de Informações de Nascidos Vivos (SINASC) em cada ano. Os dados para cada ano representam a soma de todos os registros mensais, pois a geração do dado pressupõe a conferência da data de nascimento e filiação, com a exclusão de informações duplicadas. O denominador são os recém-nascidos registrados no SINASC e, como este dado só estava disponível até 2004, o cálculo do indicador foi realizado até este ano. 


\section{Análise dos dados}

O estudo foi realizado com dados secundários provenientes do SIAB, SINASC, Censo Demográfico 2000 e estimativas populacionais intercensitárias provenientes do IBGE, disponibilizados pelo Ministério da Saúde pela Internet (http:// www.datasus.gov.br).

Para testar a adequação do indicador de cobertura do PSF foram analisados os graus de correlação e de concordância deste indicador com os indicadores de cadastramento e acompanhamento da população usuária do programa (percentual da população cadastrada no SIAB do total da população, percentual de famílias acompanhadas pelas equipes do total estimado de famílias e percentual de nascidos vivos informados no SIAB do total registrado pelo SINASC).

Foi calculado o coeficiente de correlação de Pearson para quantificar a relação entre a cobertura do PSF e os indicadores de cadastro e registro das famílias e população acompanhada pelas equipes, testando a hipótese nula que o coeficiente é igual a $0\left(\mathrm{H}_{0}: \mathrm{r}=0\right)$ versus a hipótese de trabalho que o coeficiente é diferente de zero $\left(\mathrm{H}_{\mathrm{i}}: \mathrm{r} \neq 0\right)$.

Foi avaliada a concordância entre as medidas através do coeficiente de concordância de Lin e do coeficiente de regressão estimado pelo modelo de regressão de Deming.

O coeficiente de concordância de Lin é um teste que combina uma medida de precisão (coeficiente de correlação de Pearson) com uma medida de acurácia $\left(\mathrm{C}_{\mathrm{b}}\right)$, para determinar quanto os dados observados afastam-se da linha de identidade (isto é, a linha de $45^{\circ}$ ), variando em função da distância dos dados à linha (a acurácia dos dados) e da dispersão dos dados em torno da linha (a precisão dos dados). Foi utilizada a transformação tangente hiperbólica inversa (transformação Z) que é mais robusta em relação às propriedades estatísticas, mesmo para pequenas amostras 5 .

A regressão estimada pelo modelo de Deming, ao contrário do método tradicional dos mínimos quadrados, leva em conta a existência de erro aleatório de medida nas variáveis dependente e independente, sendo apropriado para a utilização nos estudos de concordância de métodos. O método é usado para testar duas hipóteses: a de intercepto igual a 0 e a de inclinação da reta igual a 1 , sendo utilizado o intervalo de confiança a 95\% (IC95\%) para os dois parâmetros. Se as duas hipóteses são aceitas, os intervalos de confiança contêm, respectivamente, o valor 0 para o intercepto e o valor 1 para a inclinação. Se o intercepto é diferente de 0 significa que os dois métodos diferem em uma quantidade constante.
Se a inclinação da reta é diferente de 1, concluise que há uma diferença proporcional entre os dois métodos (MedCalc Software; http://www. medcalc.br/manual/deming.php).

Foi utilizado o pacote estatístico Stata 9.1 (Stata Corp., College Station, Estados Unidos). A análise foi realizada para o conjunto dos municípios e estratificada por porte populacional dos municípios (municípios com menos de 100 mil habitantes e municípios com 100 mil habitantes e mais), utilizando a população de 2000. Optouse pela definição dos estratos populacionais que vem sendo adotada pelo gestor federal do SUS para alocação de recursos municipais para o PSF, a exemplo do Projeto de Expansão e Consolidação do Saúde da Família (PROESF).

\section{Resultados}

De 1998 a 2006, o percentual de municípios brasileiros com o PSF em funcionamento aumentou de 20,4\% (1.134 municípios) para 91,8\% (5.106 municípios), com uma constante e expressiva elevação das médias anuais das coberturas municipais, que variaram de $46,8 \%$ a $80,3 \%$ e aumento do percentual de população cadastrada, das famílias acompanhadas e do registro de nascidos vivos no SIAB pelas equipes de saúde da família (Figura 1).

Observou-se um significativo aumento da correlação entre a cobertura do PSF e os três indicadores estudados. O coeficiente de correlação de Pearson entre a cobertura e o percentual da população cadastrada apresentou o maior incremento, passando de 0,35 , em 1998, para 0,81 , em 2006. No mesmo período, a correlação da cobertura do PSF com o percentual de famílias acompanhadas aumentou de 0,51 para 0,85 , e com o percentual de nascidos vivos registrados, de 0,37 para 0,78 , no período de 1998 a 2004 (Tabela 1).

Entre os municípios menores de $100 \mathrm{mil} \mathrm{ha-}$ bitantes, os coeficientes de correlação foram de magnitude ligeiramente inferior aos observados para o conjunto dos municípios, ao final do período. Em 2006, foram de 0,79 e de 0,84, respectivamente, para a correlação entre a cobertura do PSF com o percentual da população cadastrada e com o percentual de famílias acompanhadas; e, em 2004, de 0,76 para a correlação entre a cobertura do PSF e o percentual de nascidos vivos registrados. Os municípios com 100 mil ou mais habitantes apresentaram coeficientes mais altos já no ano de 2000, e para os três indicadores, a correlação com a cobertura do PSF alcançou valores em torno de 0,9 ao final do período $(0,9 ; 0,89$ e 0,87 ; respectivamente). Todos os resultados ob- 
Número de municípios, média da cobertura do Programa Saúde da Família (PSF) e percentual da população cadastrada, percentual de famílias acompanhadas e percentual de nascidos vivos registrados. Brasil, 1998 a 2006.

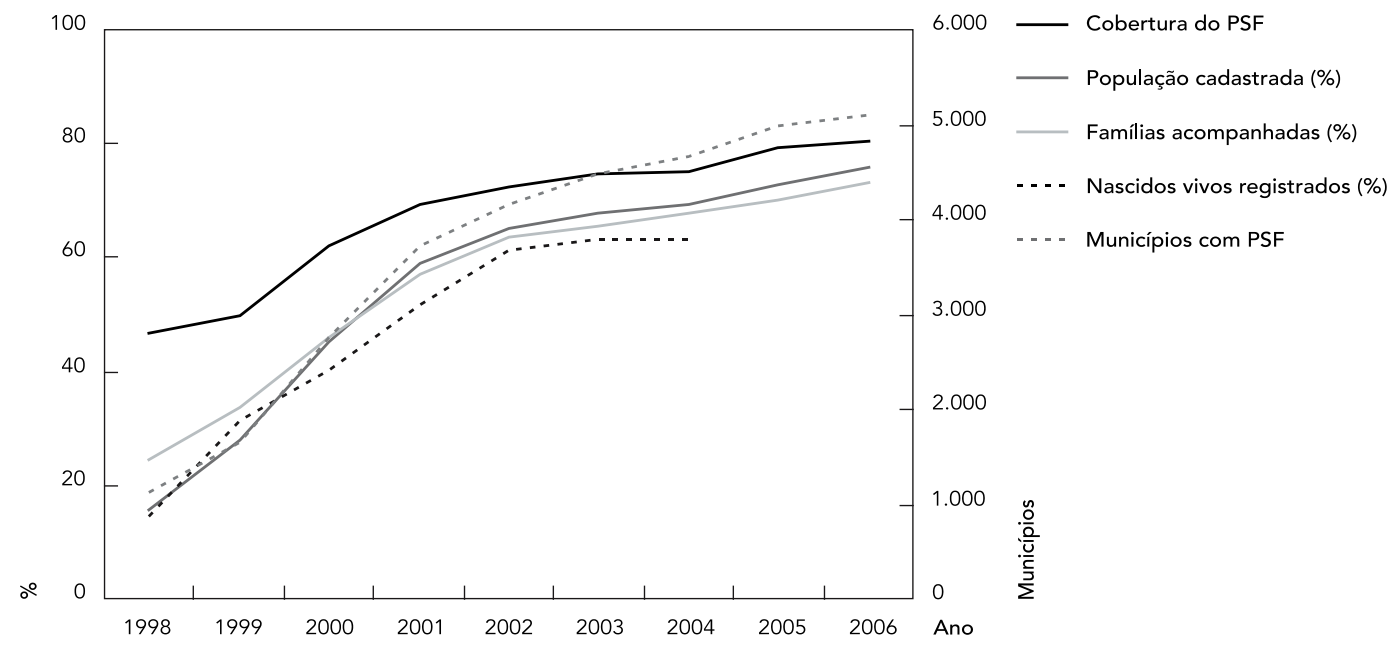

servados foram estatisticamente significantes (Tabela 1).

A análise, utilizando o coeficiente de Lin, demonstrou concordâncias positivas, ascendentes no decorrer do tempo e estatisticamente significantes, entre a cobertura e os três indicadores, com o mesmo padrão de incremento já observado para os maiores municípios. A concordância mensurada entre a cobertura do PSF e o percentual da população cadastrada variou, de 0,22 a 0,80 , e o percentual de famílias acompanhadas, de 0,39 a 0,82 , entre 1998 a 2006, e com o percentual de nascidos vivos registrados, de 0,20 a 0,72 no período de 1998 a 2004. Resultados muito semelhantes foram obtidos para os municípios menores de 100 mil habitantes. Para os maiores municípios, a concordância entre a cobertura do PSF variou de 0,24 a 0,90 , com o percentual da população cadastrada, de 0,50 a 0,86 , com o percentual de famílias acompanhadas e de 0,27 a 0,77 , com o percentual de nascidos vivos registrados, no mesmo período (Tabela 2 ).

Para o conjunto dos municípios, a regressão pelo método de Deming demonstrou que nenhum dos modelos apresentou intercepto igual a 0 ou inclinação igual a 1 , ou seja, as retas de regressão obtidas não corresponderam à linha de identidade, que expressa a perfeita concordância entre os indicadores. Entretanto, com o passar do tempo, as retas de regressão da cobertura do PSF e cada um dos três indicadores apresentaram uma incontestável tendência à aproximação dos valores dos coeficientes observados aos valores esperados (valores próximos a 1), especialmente quanto à inclinação da reta de regressão entre cobertura do PSF e percentual de famílias acompanhadas. O intercepto variou de 39,4 para 10,6, na regressão da cobertura do PSF com o percentual da população cadastrada, de 28,1 a 10,0, com o percentual de famílias acompanhadas, e de 37,9 a 17,7 , com o percentual de nascidos vivos registrados. Os valores da inclinação da reta aumentaram de 0,47 para 0,92 na regressão da cobertura do PSF com o percentual da população cadastrada, 0,76 a 0,96 , com o percentual de famílias acompanhadas, e de 0,62 a 0,91, com o percentual de nascidos vivos registrados (Tabelas 3 e 4 ).

$\mathrm{Na}$ análise por porte dos municípios, os interceptos e as inclinações das retas de regressão dos municípios menores de 100 mil habitantes apresentaram resultados bem semelhantes aos observados para o conjunto dos municípios. Os municípios com 100 mil ou mais habitantes, por outro lado, apresentaram parâmetros de regressão que revelam maior concordância entre a cobertura do PSF e o percentual da população cadastrada, o percentual de famílias acompanhadas e o percentual de nascidos vivos registrados. Os interceptos variaram de 9,7 para 2,1 na regressão da cobertura do PSF com o percentual 
Coeficiente de Pearson da correlação entre os indicadores de cobertura do Programa Saúde da Família (PSF) e o percentual da população cadastrada no Sistema de Informação da Atenção Básica (SIAB), percentual de famílias acompanhadas e percentual de nascidos vivos registrados no SIAB do total registrado no Sistema de Informações de Nascidos Vivos (SINASC), segundo porte populacional dos municípios. Brasil, 1998 a 2006.

\begin{tabular}{|c|c|c|c|c|c|c|c|c|c|}
\hline \multirow[t]{3}{*}{ Ano } & \multicolumn{9}{|c|}{ Coeficiente de correlação de Pearson (IC95\%) } \\
\hline & \multicolumn{3}{|c|}{ Total dos municípios } & \multicolumn{3}{|c|}{ Municípios $<100$ mil habitantes } & \multicolumn{3}{|c|}{ Municípios $\geq 100$ mil habitantes } \\
\hline & $\begin{array}{l}\text { População } \\
\text { cadastrada }\end{array}$ & $\begin{array}{c}\text { Famílias } \\
\text { acompanhadas }\end{array}$ & $\begin{array}{l}\text { Nascidos vivos } \\
\text { registrados }\end{array}$ & $\begin{array}{l}\text { População } \\
\text { cadastrada }\end{array}$ & $\begin{array}{c}\text { Famílias } \\
\text { acompanhadas }\end{array}$ & $\begin{array}{l}\text { Nascidos vivos } \\
\text { registrados }\end{array}$ & $\begin{array}{l}\text { População } \\
\text { cadastrada }\end{array}$ & $\begin{array}{c}\text { Famílias } \\
\text { acompanhadas }\end{array}$ & $\begin{array}{c}\text { Nascidos vivos } \\
\text { registrados }\end{array}$ \\
\hline 1998 & $\begin{array}{c}0,35 \\
(0,30-0,40)\end{array}$ & $\begin{array}{c}0,51 \\
(0,46-0,55)\end{array}$ & $\begin{array}{c}0,37 \\
(0,32-0,42)\end{array}$ & $\begin{array}{c}0,32 \\
(0,26-0,37)\end{array}$ & $\begin{array}{c}0,47 \\
(0,42-0,52)\end{array}$ & $\begin{array}{c}0,33 \\
(0,28-0,39)\end{array}$ & $\begin{array}{c}0,37 \\
(0,19-0,53)\end{array}$ & $\begin{array}{c}0,55 \\
(0,40-0,67)\end{array}$ & $\begin{array}{c}0,60 \\
(0,45-0,71)\end{array}$ \\
\hline 1999 & $\begin{array}{c}0,47 \\
(0,43-0,50)\end{array}$ & $\begin{array}{c}0,60 \\
(0,57-0,63)\end{array}$ & $\begin{array}{c}0,50 \\
(0,47-0,54)\end{array}$ & $\begin{array}{c}0,44 \\
(0,39-0,48)\end{array}$ & $\begin{array}{c}0,58 \\
(0,54-0,61)\end{array}$ & $\begin{array}{c}0,47 \\
(0,43-0,51)\end{array}$ & $\begin{array}{c}0,71 \\
(0,60-0,79)\end{array}$ & $\begin{array}{c}0,67 \\
(0,56-0,76)\end{array}$ & $\begin{array}{c}0,75 \\
(0,66-0,82)\end{array}$ \\
\hline 2000 & $\begin{array}{c}0,60 \\
(0,57-0,62)\end{array}$ & $\begin{array}{c}0,70 \\
(0,68-0,72)\end{array}$ & $\begin{array}{c}0,53 \\
(0,50-0,56)\end{array}$ & $\begin{array}{c}0,57 \\
(0,54-0,59)\end{array}$ & $\begin{array}{c}0,67 \\
(0,65-0,69)\end{array}$ & $\begin{array}{c}0,50 \\
(0,47-0,52)\end{array}$ & $\begin{array}{c}0,87 \\
(0,83-0,90)\end{array}$ & $\begin{array}{c}0,85 \\
(0,80-0,89)\end{array}$ & $\begin{array}{c}0,81 \\
(0,74-0,86)\end{array}$ \\
\hline 2001 & $\begin{array}{c}0,69 \\
(0,67-0,71)\end{array}$ & $\begin{array}{c}0,82 \\
(0,81-0,83)\end{array}$ & $\begin{array}{c}0,65 \\
(0,63-0,66)\end{array}$ & $\begin{array}{c}0,66 \\
(0,64-0,68)\end{array}$ & $\begin{array}{c}0,80 \\
(0,79-0,82)\end{array}$ & $\begin{array}{c}0,61 \\
(0,59-0,63)\end{array}$ & $\begin{array}{c}0,83 \\
(0,77-0,87)\end{array}$ & $\begin{array}{c}0,86 \\
(0,81-0,89)\end{array}$ & $\begin{array}{c}0,79 \\
(0.73-0,84)\end{array}$ \\
\hline 2002 & $\begin{array}{c}0,74 \\
(0,73-0,75)\end{array}$ & $\begin{array}{c}0,84 \\
(0,83-0,85)\end{array}$ & $\begin{array}{c}0,77 \\
(0,75-0,78)\end{array}$ & $\begin{array}{c}0,71 \\
(0,70-0,73)\end{array}$ & $\begin{array}{c}0,83 \\
(0,82-0,84)\end{array}$ & $\begin{array}{c}0,74 \\
(0,73-0,76)\end{array}$ & $\begin{array}{c}0,85 \\
(0,80-0,88)\end{array}$ & $\begin{array}{c}0,91 \\
(0,88-0,93)\end{array}$ & $\begin{array}{c}0,85 \\
(0,81-0,89)\end{array}$ \\
\hline 2003 & $\begin{array}{c}0,77 \\
(0,76-0,78)\end{array}$ & $\begin{array}{c}0,85 \\
(0,84-0,85)\end{array}$ & $\begin{array}{c}0,79 \\
(0,78-0,80)\end{array}$ & $\begin{array}{c}0,75 \\
(0,73-0,76)\end{array}$ & $\begin{array}{c}0,83 \\
(0,82-0,84)\end{array}$ & $\begin{array}{c}0,76 \\
(0,75-0,78)\end{array}$ & $\begin{array}{c}0,87 \\
(0,83-0,90)\end{array}$ & $\begin{array}{c}0,92 \\
(0,89-0,94)\end{array}$ & $\begin{array}{c}0,87 \\
(0,83-0,90)\end{array}$ \\
\hline 2004 & $\begin{array}{c}0,77 \\
(0,76-0,78)\end{array}$ & $\begin{array}{c}0,84 \\
(0,83-0,85)\end{array}$ & $\begin{array}{c}0,78 \\
(0,77-0,79)\end{array}$ & $\begin{array}{c}0,75 \\
(0,73-0,76)\end{array}$ & $\begin{array}{c}0,82 \\
(0,81-0,83)\end{array}$ & $\begin{array}{c}0,76 \\
(0,75-0,77)\end{array}$ & $\begin{array}{c}0,91 \\
(0,88-0,93)\end{array}$ & $\begin{array}{c}0,92 \\
(0,90-0,94)\end{array}$ & - \\
\hline 2005 & $\begin{array}{c}0,80 \\
(0,78-0,80)\end{array}$ & $\begin{array}{c}0,85 \\
(0,84-0,86)\end{array}$ & - & $\begin{array}{c}0,77 \\
(0,76-0,78)\end{array}$ & $\begin{array}{c}0,83 \\
(0,82-0,84)\end{array}$ & - & $\begin{array}{c}0,87 \\
(0,84-0,90)\end{array}$ & $\begin{array}{c}0,91 \\
(0,88-0,93)\end{array}$ & - \\
\hline 2006 & $\begin{array}{c}0,81 \\
(0,80-0,82)\end{array}$ & $\begin{array}{c}0,85 \\
(0,84-0,86)\end{array}$ & - & $\begin{array}{c}0,79 \\
(0,77-0,80)\end{array}$ & $\begin{array}{c}0,84 \\
(0,83-0,84)\end{array}$ & - & $\begin{array}{c}0,90 \\
(0,87-0,92)\end{array}$ & $\begin{array}{c}0,89 \\
(0,85-0,91)\end{array}$ & - \\
\hline
\end{tabular}

da população cadastrada, de 7,6 a 4,6, com o percentual de famílias acompanhadas, e de 6,6 a 6,2 , com o percentual de nascidos vivos registrados. Para os maiores municípios, os valores dos intervalos de confiança da inclinação da reta da cobertura do PSF com o percentual da população cadastradas incluíram o valor 1 em todos os anos; com o percentual de famílias acompanhadas, o valor 1 só não foi incluído nos anos 2000 e 2002 e para o percentual de nascidos vivos registrados, o valor 1 foi incluído apenas nos anos de 1999 e 2000 (Tabelas 3 e 4).

\section{Discussão}

O estudo demonstrou que, no período inicial da expansão do PSF, o indicador de cobertura utilizado não expressava a magnitude da população beneficiária do programa. Paulatinamente, com a sua consolidação, em especial a partir de 2000, o indicador passou a se constituir, cada vez mais, em uma boa estimativa da cobertura efetiva do PSF, apresentando valores semelhantes aos referentes à população cadastrada, ao número de famílias acompanhadas e ao número de nascidos vivos registrados, segundo as informações alimentadas pelas próprias equipes de saúde da família no SIAB em cada município. Esta evolução foi diferenciada segundo o porte dos municípios, ocorrendo de forma antecipada e mais expressiva entre os maiores municípios (com 100 mil ou mais habitantes).

Antes da discussão destes achados, faz-se necessário explicitar algumas questões que orientaram as opções metodológicas adotadas. Primeiro, o estudo realizado consistiu na avaliação de concordância, e não na calibração das medidas, isto porque não existia, e ainda não existe, um indicador de cobertura do PSF que represente um padrão-ouro para uma avaliação de âmbito nacional. Só podemos optar pela calibração quando o resultado obtido pelo método a ser testado é comparado com o "verdadeiro" valor, obtido por um método de elevada acurácia 6 .

Em se tratando de um estudo desta natureza temos que considerar o grande debate, especialmente no campo da estatística, acerca da avaliação da concordância de métodos, em especial quanto ao uso dos coeficientes de correlação e do 
Coeficiente de concordância de Lin dos indicadores de cobertura do Programa Saúde da Família (PSF) e o percentual da população cadastrada no Sistema de Informação da Atenção Básica (SIAB), percentual de famílias acompanhadas e percentual de nascidos vivos registrados no SIAB do total registrado no Sistema de Informações de Nascidos Vivos (SINASC). Brasil, 1998 a 2006.

\begin{tabular}{|c|c|c|c|c|c|c|c|c|c|}
\hline \multirow[t]{3}{*}{ Ano } & \multicolumn{9}{|c|}{ Coeficiente de concordância de Lin (IC95\%) } \\
\hline & \multicolumn{3}{|c|}{ Total dos municípios } & \multicolumn{3}{|c|}{ Municípios < 100 mil habitantes } & \multicolumn{3}{|c|}{ Municípios $\geq 100$ mil habitantes } \\
\hline & $\begin{array}{l}\text { População } \\
\text { cadastrada }\end{array}$ & $\begin{array}{c}\text { Famílias } \\
\text { acompanhadas }\end{array}$ & $\begin{array}{l}\text { Nascidos vivos } \\
\text { registrados }\end{array}$ & $\begin{array}{l}\text { População } \\
\text { cadastrada }\end{array}$ & $\begin{array}{c}\text { Famílias } \\
\text { acompanhadas }\end{array}$ & $\begin{array}{l}\text { Nascidos vivos } \\
\text { registrados }\end{array}$ & $\begin{array}{l}\text { População } \\
\text { cadastrada }\end{array}$ & $\begin{array}{c}\text { Famílias } \\
\text { acompanhadas }\end{array}$ & $\begin{array}{c}\text { Nascidos vivos } \\
\text { registrados }\end{array}$ \\
\hline 1998 & $\begin{array}{c}0,22 \\
(0,18-0,25)\end{array}$ & $\begin{array}{c}0,39 \\
(0,35-0,43)\end{array}$ & $\begin{array}{c}0,20 \\
(0,17-0,23)\end{array}$ & $\begin{array}{c}0,19 \\
(0,15-0,22)\end{array}$ & $\begin{array}{c}0,35 \\
(0,31-0,39)\end{array}$ & $\begin{array}{c}0,17 \\
(0,14-0,20)\end{array}$ & $\begin{array}{c}0,24 \\
(0,12-0,35)\end{array}$ & $\begin{array}{c}0,50 \\
(0,36-0,62)\end{array}$ & $\begin{array}{c}0,27 \\
(0,18-0,34)\end{array}$ \\
\hline 1999 & $\begin{array}{c}0,37 \\
(0,34-0,41)\end{array}$ & $\begin{array}{c}0,53 \\
(0,49-0,56)\end{array}$ & $\begin{array}{c}0,43 \\
(0,40-0,47)\end{array}$ & $\begin{array}{c}0,34 \\
(0,31-0,38)\end{array}$ & $\begin{array}{c}0,49 \\
(0,46-0,53)\end{array}$ & $\begin{array}{c}0,40 \\
(0,36-0,43)\end{array}$ & $\begin{array}{c}0,65 \\
(0,54-0,74)\end{array}$ & $\begin{array}{c}0,65 \\
(0,54-0,74)\end{array}$ & $\begin{array}{c}0,67 \\
(0,58-0,75)\end{array}$ \\
\hline 2000 & $\begin{array}{c}0,53 \\
(0,50-0,55)\end{array}$ & $\begin{array}{c}0,61 \\
(0,59-0,63)\end{array}$ & $\begin{array}{c}0,43 \\
(0,40-0,45)\end{array}$ & $\begin{array}{c}0,49 \\
(0,46-0,51)\end{array}$ & $\begin{array}{c}0,58 \\
(0,55-0,60)\end{array}$ & $\begin{array}{c}0,39 \\
(0,36-0,42)\end{array}$ & $\begin{array}{c}0,84 \\
(0,79-0,88)\end{array}$ & $\begin{array}{c}0,78 \\
(0,72-0,83)\end{array}$ & $\begin{array}{c}0,71 \\
(0,64-0,77)\end{array}$ \\
\hline 2001 & $\begin{array}{c}0,65 \\
(0,63-0,67)\end{array}$ & $\begin{array}{c}0,76 \\
(0,74-0,77)\end{array}$ & $\begin{array}{c}0,55 \\
(0,53-0,57)\end{array}$ & $\begin{array}{c}0,62 \\
(0,60-0,64)\end{array}$ & $\begin{array}{c}0,73 \\
(0,72-0,75)\end{array}$ & $\begin{array}{c}0,52 \\
(0,50-0,54)\end{array}$ & $\begin{array}{c}0,82 \\
(0,76-0,86)\end{array}$ & $\begin{array}{c}0,82 \\
(0,77-0,86)\end{array}$ & $\begin{array}{c}0,71 \\
(0,63-0,77)\end{array}$ \\
\hline 2002 & $\begin{array}{c}0,72 \\
(0,70-0,73)\end{array}$ & $\begin{array}{c}0,81 \\
(0,80-0,82)\end{array}$ & $\begin{array}{c}0,72 \\
(0,70-0,73)\end{array}$ & $\begin{array}{c}0,69 \\
(0,67-0,70)\end{array}$ & $\begin{array}{c}0,79 \\
(0,78-0,80)\end{array}$ & $\begin{array}{c}0,69 \\
(0,67-0,70)\end{array}$ & $\begin{array}{c}0,84 \\
(0,79-0,88)\end{array}$ & $\begin{array}{c}0,88 \\
(0,85-0,91)\end{array}$ & $\begin{array}{c}0,77 \\
(0,71-0,81)\end{array}$ \\
\hline 2003 & $\begin{array}{c}0,75 \\
(0,73-0,76)\end{array}$ & $\begin{array}{c}0,81 \\
(0,81-0,82)\end{array}$ & $\begin{array}{c}0,73 \\
(0,72-0,74)\end{array}$ & $\begin{array}{c}0,72 \\
(0,71-0,73)\end{array}$ & $\begin{array}{c}0,79 \\
(0,78-0,80)\end{array}$ & $\begin{array}{c}0,71 \\
(0,69-0,72)\end{array}$ & $\begin{array}{c}0,87 \\
(0,83-0,90)\end{array}$ & $\begin{array}{c}0,89 \\
(0,86-0,92)\end{array}$ & $\begin{array}{c}0,77 \\
(0,72-0,82)\end{array}$ \\
\hline 2004 & $\begin{array}{c}0,75 \\
(0,74-0,77)\end{array}$ & $\begin{array}{c}0,81 \\
(0,81-0,82)\end{array}$ & $\begin{array}{c}0,72 \\
(0,71-0,73)\end{array}$ & $\begin{array}{c}0,73 \\
(0,71-0,74)\end{array}$ & $\begin{array}{c}0,79 \\
(0,78-0,80)\end{array}$ & $\begin{array}{c}0,70 \\
(0,68-0,71)\end{array}$ & $\begin{array}{c}0,90 \\
(0,88-0,93)\end{array}$ & $\begin{array}{c}0,89 \\
(0,86-0,92)\end{array}$ & $\begin{array}{c}0,77 \\
(0,72-0,81)\end{array}$ \\
\hline 2005 & $\begin{array}{c}0,77 \\
(0,76-0,78)\end{array}$ & $\begin{array}{c}0,80 \\
(0,80-0,81)\end{array}$ & - & $\begin{array}{c}0,75 \\
(0,73-0,76)\end{array}$ & $\begin{array}{c}0,78 \\
(0,77-0,79)\end{array}$ & - & $\begin{array}{c}0,86 \\
(0,82-0,89)\end{array}$ & $\begin{array}{c}0,86 \\
(0,83-0,89)\end{array}$ & - \\
\hline 2006 & $\begin{array}{c}0,80 \\
(0,79-0,81)\end{array}$ & $\begin{array}{c}0,82 \\
(0,81-0,83)\end{array}$ & - & $\begin{array}{c}0,77 \\
(0,76-0,78)\end{array}$ & $\begin{array}{c}0,81 \\
(0,80-0,82)\end{array}$ & - & $\begin{array}{c}0,90 \\
(0,87-0,92)\end{array}$ & $\begin{array}{c}0,86 \\
(0,82-0,89)\end{array}$ & - \\
\hline
\end{tabular}

método de regressão dos mínimos quadrados. O uso freqüente de coeficientes de correlação neste tipo de estudo tem sido questionado porque a correlação mede a força da relação linear entre duas variáveis e não a concordância, ou seja, o seu valor pode ser aproximadamente 1 (ou igual a 1) mesmo quando há variação considerável entre os dois métodos. Além disso, a correlação, ao contrário da concordância, não é afetada pela escala de medida utilizada; a correlação é afetada pela variação do "verdadeiro" valor da medida na amostra, sendo maior em amostras mais heterogêneas e, finalmente, o teste de significância usual (hipótese nula de $\mathrm{r}=0$ ) é irrelevante para comparação de métodos, não bastando demonstrar que dois métodos designados para medir a mesma quantidade estão relacionados 7. Quanto à utilização da regressão linear pelo método dos mínimos quadrados, o pressuposto de que os valores da variável independente $(\mathrm{X})$ podem, pelo menos para efeitos práticos, ser considerados como livres de erro, só poderia ser assumido em estudo em que um dos métodos fosse considerado como padrão-ouro, limitando o seu uso em estudos de concordância 8,9.
Estas questões determinaram a decisão de agregar à medida da correlação, de corrente interpretação na área de saúde pública, outros métodos que buscassem corroborar os achados do estudo. Como pudemos observar, todos os métodos apresentaram uma tendência temporal ascendente de concordância entre a cobertura estimada e os três indicadores, entretanto, os resultados obtidos a partir da análise dos coeficientes de Pearson foram sistematicamente mais expressivos do que os obtidos com os demais métodos utilizados. Dadas as questões metodológicas anteriormente descritas, os resultados obtidos pelos métodos de Lin e Deming devem representar melhor os níveis de concordância entre os indicadores.

Por outro lado, a principal questão a que devemos estar atentos nesta discussão é que, como os indicadores utilizados para a avaliação do indicador de cobertura apresentam, eles próprios, erros de mensuração, convém refletir acerca das fontes de erro daqueles indicadores para correta interpretação dos resultados obtidos.

As fontes de erro do indicador de registro de nascidos vivos são provenientes do SIAB e do 
Coeficiente de regressão $\beta_{0}$ estimado pelo método Deming da associação entre o indicador de cobertura do Programa Saúde da Família (PSF) e o percentual da população cadastrada no Sistema de Informação da Atenção Básica (SIAB), de famílias acompanhadas e nascidos vivos registrados no SIAB do total registrado no Sistema de Informações de Nascidos Vivos (SINASC). Brasil, 1998 a 2006.

\begin{tabular}{|c|c|c|c|c|c|c|c|c|c|}
\hline \multirow[t]{3}{*}{ Ano } & \multicolumn{9}{|c|}{ Coeficiente de regressão $\beta_{0}$ (IC95\%) * } \\
\hline & \multicolumn{3}{|c|}{ Total dos municípios } & \multicolumn{3}{|c|}{ Municípios < 100 mil habitantes } & \multicolumn{3}{|c|}{ Municípios $\geq 100$ mil habitantes } \\
\hline & $\begin{array}{l}\text { População } \\
\text { cadastrada }\end{array}$ & $\begin{array}{c}\text { Famílias } \\
\text { acompanhadas }\end{array}$ & $\begin{array}{c}\text { Nascidos vivos } \\
\text { registrados }\end{array}$ & $\begin{array}{l}\text { População } \\
\text { cadastrada }\end{array}$ & $\begin{array}{c}\text { Famílias } \\
\text { acompanhadas }\end{array}$ & $\begin{array}{c}\text { Nascidos vivos } \\
\text { registrados }\end{array}$ & $\begin{array}{l}\text { População } \\
\text { cadastrada }\end{array}$ & $\begin{array}{c}\text { Famílias } \\
\text { acompanhadas }\end{array}$ & $\begin{array}{c}\text { Nascidos vivos } \\
\text { registrados }\end{array}$ \\
\hline 1998 & $\begin{array}{c}39,4 \\
(37,2-41,6)\end{array}$ & $\begin{array}{c}28,1 \\
(25,4-30,8)\end{array}$ & $\begin{array}{c}37,9 \\
(35,6-40,1)\end{array}$ & $\begin{array}{c}43,5 \\
(41,2-45,7)\end{array}$ & $\begin{array}{c}32,5 \\
(29,5-35,5)\end{array}$ & $\begin{array}{c}42,1 \\
(39,8-44,4)\end{array}$ & $\begin{array}{c}9,7 \\
(6,4-13,0)\end{array}$ & $\begin{array}{c}7,6 \\
(2,8-12,4)\end{array}$ & $\begin{array}{c}6,6 \\
(4,1-9,1)\end{array}$ \\
\hline 1999 & $\begin{array}{c}34,2 \\
(31,9-36,4)\end{array}$ & $\begin{array}{c}22,0 \\
(19,8-24,3)\end{array}$ & $\begin{array}{c}30,2 \\
(27,9-32,4)\end{array}$ & $\begin{array}{c}37,7 \\
(35,4-40,0)\end{array}$ & $\begin{array}{c}25,2 \\
(22,7-27,7)\end{array}$ & $\begin{array}{c}33,8 \\
(31,4-32,2)\end{array}$ & $\begin{array}{c}7,8 \\
(5,2-10,5)\end{array}$ & $\begin{array}{c}5,1 \\
(2,7-7,6)\end{array}$ & $\begin{array}{c}5,3 \\
(2,0-8,5)\end{array}$ \\
\hline 2000 & $\begin{array}{c}29,3 \\
(27,1-31,5)\end{array}$ & $\begin{array}{c}18,7 \\
(16,9-20,6)\end{array}$ & $\begin{array}{c}34,1 \\
(32,0-36,1)\end{array}$ & $\begin{array}{c}33,4 \\
(31,0-35,8)\end{array}$ & $\begin{array}{c}21,5 \\
(19,4-23,6)\end{array}$ & $\begin{array}{c}38,4 \\
(36,2-40,7)\end{array}$ & $\begin{array}{c}3,6 \\
(1,8-5,4)\end{array}$ & $\begin{array}{c}4,4 \\
(2,5-6,4)\end{array}$ & $\begin{array}{c}5,1 \\
(2,7-7,4)\end{array}$ \\
\hline 2001 & $\begin{array}{c}22,7 \\
(20,7-24,8)\end{array}$ & $\begin{array}{c}12,5 \\
(11,2-13,7)\end{array}$ & $\begin{array}{c}28,4 \\
(26,5-30,3)\end{array}$ & $\begin{array}{c}26,3 \\
(23,9-28,6)\end{array}$ & $\begin{array}{c}14,2 \\
(12,7-15,7)\end{array}$ & $\begin{array}{c}32,2 \\
(30,1-34,2)\end{array}$ & $\begin{array}{c}3,2 \\
(1,2-5,1)\end{array}$ & $\begin{array}{c}4,4 \\
(2,5-6,2)\end{array}$ & $\begin{array}{c}4,8 \\
(2,5-7,1)\end{array}$ \\
\hline 2002 & $\begin{array}{c}17,7 \\
(15,9-19,5)\end{array}$ & $\begin{array}{c}10,2 \\
(8,9-11,4)\end{array}$ & $\begin{array}{c}17,1 \\
(15,7-18,5)\end{array}$ & $\begin{array}{c}20,5 \\
(18,5-22,6)\end{array}$ & $\begin{array}{c}11,4 \\
(10,0-12,9)\end{array}$ & $\begin{array}{c}19,1 \\
(17,5-20,7)\end{array}$ & $\begin{array}{c}3,2 \\
(1,1-5,4)\end{array}$ & $\begin{array}{c}3,2 \\
(1,6-4,7)\end{array}$ & $\begin{array}{c}4,1 \\
(1,8-6,4)\end{array}$ \\
\hline 2003 & $\begin{array}{c}16,6 \\
(14,9-18,4)\end{array}$ & $\begin{array}{c}11,8 \\
(10,5-13,0)\end{array}$ & $\begin{array}{c}18,4 \\
(17,1-19,7)\end{array}$ & $\begin{array}{c}19,2 \\
(17,2-21,1)\end{array}$ & $\begin{array}{c}13,3 \\
(11,8-14,8)\end{array}$ & $\begin{array}{c}20,4 \\
(18,9-21,9)\end{array}$ & $\begin{array}{c}3,2 \\
(1,3-5,2)\end{array}$ & $\begin{array}{c}3,8 \\
(2,2-5,4)\end{array}$ & $\begin{array}{c}5,1 \\
(2,8-7,4)\end{array}$ \\
\hline 2004 & $\begin{array}{c}14,9 \\
(13,2-16,7)\end{array}$ & $\begin{array}{c}9,7 \\
(8,5-11,0)\end{array}$ & $\begin{array}{c}17,7 \\
(16,4-19,0)\end{array}$ & $\begin{array}{c}17,1 \\
(15,1-19,1)\end{array}$ & $\begin{array}{c}10,5 \\
(9,0-11,9)\end{array}$ & $\begin{array}{c}19,3 \\
(17,8-20,8)\end{array}$ & $\begin{array}{c}3,9 \\
(2,0-5,7)\end{array}$ & $\begin{array}{c}6,0 \\
(4,3-7,7)\end{array}$ & $\begin{array}{c}6,2 \\
(4,1-8,4)\end{array}$ \\
\hline 2005 & $\begin{array}{c}15,1 \\
(13,5-16,8)\end{array}$ & $\begin{array}{c}12,9 \\
(11,8-14,0)\end{array}$ & - & $\begin{array}{c}17,6 \\
(15,7-19,5)\end{array}$ & $\begin{array}{c}14,2 \\
(12,9-15,5)\end{array}$ & - & $\begin{array}{c}3,1 \\
(0,9-5,3)\end{array}$ & $\begin{array}{c}5,1 \\
(3,3-7,0)\end{array}$ & - \\
\hline 2006 & $\begin{array}{c}10,6 \\
(9,0-12,1)\end{array}$ & $\begin{array}{c}10,0 \\
(8,9-11,2)\end{array}$ & - & $\begin{array}{c}12,7 \\
(10,9-14,5)\end{array}$ & $\begin{array}{c}10,9 \\
(9,6-12,1)\end{array}$ & - & $\begin{array}{c}2,1 \\
(0,2-4,1)\end{array}$ & $\begin{array}{c}4,6 \\
(2,4-6,8)\end{array}$ & - \\
\hline
\end{tabular}

* $\beta_{0}$ (IC95\%): intercepto da reta de regressão e intervalo de confiança a 95\%.

SINASC. No caso do SINASC, embora a cobertura venha melhorando expressivamente desde a sua implantação, ainda não é universal, sendo estimada, em 2002, em 93\% para o Brasil e, em média, $75 \%$ nas regiões Norte e Nordeste 10 . No SIAB, os dados referentes aos nascimentos são registrados mensalmente pelos agentes comunitários de saúde, a partir das informações coletadas em visitas domiciliares, não existindo estimativas nacionais de sub-registro desse sistema. Há indícios de que não existe uma coincidência total das áreas cobertas do SIAB e SINASC, o que tem, inclusive, desencadeado iniciativas do Ministério da Saúde de integração dos dois sistemas e do Sistema de Informações sobre Mortalidade (SIM), desde 2001, para o aperfeiçoamento da cobertura e da qualidade da informação de nascimentos e óbitos, em populações assistidas pelas equipes de saúde da família 11 .

Quanto aos indicadores de percentual da população cadastrada e de famílias acompanhadas, devem ser acrescidas às fontes de erro do SIAB - incluindo a ausência de orientações para atualização do cadastro de famílias - as distorções nas estimativas populacionais inerentes aos denominadores utilizados. As projeções de população, dada a quantidade de variáveis que precisam ser consideradas na formulação de hipóteses de crescimento populacional, são suscetíveis a erros, em especial quando se referem a pequenas áreas, onde é maior a variabilidade das variáveis que regem a dinâmica demográfica e são maiores os problemas quanto à qualidade nos registros dos eventos populacionais 12 .

Temos que ressaltar, ainda, que nenhum dos três indicadores usados para comparação com a cobertura do PSF foi criado, ou vem sendo utilizado, com esta finalidade. Ressalta-se que, no caso dos nascidos vivos, a interpretação dos resultados deve ser especialmente cuidadosa, uma vez que, representando uma parcela muito restrita da população usuária, não seria esperada uma estrita ou direta correspondência entre este indicador e a cobertura do programa. Além disso, a ampliação da cobertura do PSF, do cadastramento e do registro da população adstrita são efeitos esperados da consolidação do programa nos municípios, intrinsecamente relacionados 
Coeficiente de regressão $\beta_{1}$ estimado pelo método Deming da associação entre o indicador de cobertura do Programa Saúde da Família (PSF) e o percentual da população cadastrada no Sistema de Informação da Atenção Básica (SIAB), de famílias acompanhadas e nascidos vivos registrados no SIAB do total registrado no Sistema de Informações de Nascidos Vivos (SINASC). Brasil, 1998 a 2006.

\begin{tabular}{|c|c|c|c|c|c|c|c|c|c|}
\hline \multirow[t]{3}{*}{ Ano } & \multicolumn{9}{|c|}{ Coeficiente de regressão $\beta_{1}$ (IC95\%) * } \\
\hline & \multicolumn{3}{|c|}{ Total dos municípios } & \multicolumn{3}{|c|}{ Municípios $<100$ mil habitantes } & \multicolumn{3}{|c|}{ Municípios $\geq 100$ mil habitantes } \\
\hline & $\begin{array}{l}\text { População } \\
\text { cadastrada }\end{array}$ & $\begin{array}{c}\text { Famílias } \\
\text { acompanhadas }\end{array}$ & $\begin{array}{l}\text { Nascidos vivos } \\
\text { registrados }\end{array}$ & $\begin{array}{l}\text { População } \\
\text { cadastrada }\end{array}$ & $\begin{array}{c}\text { Famílias } \\
\text { acompanhadas }\end{array}$ & $\begin{array}{l}\text { Nascidos vivos } \\
\text { registrados }\end{array}$ & $\begin{array}{l}\text { População } \\
\text { cadastrada }\end{array}$ & $\begin{array}{c}\text { Famílias } \\
\text { acompanhadas }\end{array}$ & $\begin{array}{c}\text { Nascidos vivos } \\
\text { registrados }\end{array}$ \\
\hline 1998 & $\begin{array}{c}0,47 \\
(0,41-0,53)\end{array}$ & $\begin{array}{c}0,76 \\
(0,69-0,82)\end{array}$ & $\begin{array}{c}0,62 \\
(0,53-0,70)\end{array}$ & $\begin{array}{c}0,40 \\
(0,33-0,46)\end{array}$ & $\begin{array}{c}0,67 \\
(0,60-0,73)\end{array}$ & $\begin{array}{c}0,51 \\
(0,44-0,59)\end{array}$ & $\begin{array}{c}0,89 \\
(0,53-1,26)\end{array}$ & $\begin{array}{c}0,77 \\
(-0,21-1,75)\end{array}$ & $\begin{array}{c}2,21 \\
(1,88-2,53)\end{array}$ \\
\hline 1999 & $\begin{array}{c}0,56 \\
(0,52-0,61)\end{array}$ & $\begin{array}{c}0,83 \\
(0,78-0,87)\end{array}$ & $\begin{array}{c}0,62 \\
(0,58-0,67)\end{array}$ & $\begin{array}{c}0,50 \\
(0,45-0,55)\end{array}$ & $\begin{array}{c}0,77 \\
(0,73-0,82)\end{array}$ & $\begin{array}{c}0,56 \\
(0,51-0,61)\end{array}$ & $\begin{array}{c}0,89 \\
(0,61-1,17)\end{array}$ & $\begin{array}{c}0,91 \\
(0,65-1,16)\end{array}$ & $\begin{array}{c}1,15 \\
(0,71-1,58)\end{array}$ \\
\hline 2000 & $\begin{array}{c}0,73 \\
(0,70-0,76)\end{array}$ & $\begin{array}{c}0,95 \\
(0,92-0,98)\end{array}$ & $\begin{array}{c}0,70 \\
(0,67-0,74)\end{array}$ & $\begin{array}{c}0,67 \\
(0,63-0,70)\end{array}$ & $\begin{array}{c}0,91 \\
(0,87-0,94)\end{array}$ & $\begin{array}{c}0,63 \\
(0,59-0,67)\end{array}$ & $\begin{array}{c}1,07 \\
(0,94-1,20)\end{array}$ & $\begin{array}{c}1,18 \\
(1,01-1,34)\end{array}$ & $\begin{array}{c}1,22 \\
(1,00-1,45)\end{array}$ \\
\hline 2001 & $\begin{array}{c}0,79 \\
(0,77-0,82)\end{array}$ & $\begin{array}{c}1,00 \\
(0,98-1,02)\end{array}$ & $\begin{array}{c}0,80 \\
(0,77-0,82)\end{array}$ & $\begin{array}{c}0,75 \\
(0,72-0,78)\end{array}$ & $\begin{array}{c}0,98 \\
(0,96-1,00)\end{array}$ & $\begin{array}{c}0,74 \\
(0,71-0,77)\end{array}$ & $\begin{array}{c}0,97 \\
(0,88-1,06)\end{array}$ & $\begin{array}{c}1,07 \\
(0,96-1,18)\end{array}$ & $\begin{array}{c}1,20 \\
(1,04-1,36)\end{array}$ \\
\hline 2002 & $\begin{array}{c}0,84 \\
(0,82-0,87)\end{array}$ & $\begin{array}{c}0,98 \\
(0,97-1,00)\end{array}$ & $\begin{array}{c}0,90 \\
(0,89-0,92)\end{array}$ & $\begin{array}{c}0,81 \\
(0,78-0,83)\end{array}$ & $\begin{array}{c}0,96 \\
(0,95-0,98)\end{array}$ & $\begin{array}{c}0,88 \\
(0,86-0,90)\end{array}$ & $\begin{array}{c}0,97 \\
(0,89-1,04)\end{array}$ & $\begin{array}{c}1,09 \\
(1,02-1,16)\end{array}$ & $\begin{array}{c}1,22 \\
(1,09-1,36)\end{array}$ \\
\hline 2003 & $\begin{array}{c}0,85 \\
(0,83-0,87)\end{array}$ & $\begin{array}{c}0,96 \\
(0,94-0,97)\end{array}$ & $\begin{array}{c}0,89 \\
(0,88-0,91)\end{array}$ & $\begin{array}{c}0,82 \\
(0,80-0,85)\end{array}$ & $\begin{array}{c}0,94 \\
(0,92-0,96)\end{array}$ & $\begin{array}{c}0,86 \\
(0,85-0,88)\end{array}$ & $\begin{array}{c}0,97 \\
(0,91-1,03)\end{array}$ & $\begin{array}{c}1,05 \\
(0,99-1,11)\end{array}$ & $\begin{array}{c}1,20 \\
(1,08-1,33)\end{array}$ \\
\hline 2004 & $\begin{array}{c}0,87 \\
(0,85-0,89)\end{array}$ & $\begin{array}{c}0,97 \\
(0,95-0,98)\end{array}$ & $\begin{array}{c}0,91 \\
(0,89-0,93)\end{array}$ & $\begin{array}{c}0,84 \\
(0,82-0,86)\end{array}$ & $\begin{array}{c}0,96 \\
(0,94-0,97)\end{array}$ & $\begin{array}{c}0,89 \\
(0,87-0,91)\end{array}$ & $\begin{array}{c}0,96 \\
(0,90-1,02)\end{array}$ & $\begin{array}{c}1,01 \\
(0,95-1,07)\end{array}$ & $\begin{array}{c}1,20 \\
(1,10-1,31)\end{array}$ \\
\hline 2005 & $\begin{array}{c}0,88 \\
(0,86-0,90)\end{array}$ & $\begin{array}{c}0,95 \\
(0,93-0,96)\end{array}$ & - & $\begin{array}{c}0,85 \\
(0,83-0,87)\end{array}$ & $\begin{array}{c}0,93 \\
(0,92-0,95)\end{array}$ & - & $\begin{array}{c}1,01 \\
(0,93-1,09)\end{array}$ & $\begin{array}{c}1,07 \\
(1,00-1,14)\end{array}$ & - \\
\hline 2006 & $\begin{array}{c}0,92 \\
(0,90-0,94)\end{array}$ & $\begin{array}{c}0,96 \\
(0,95-0,97)\end{array}$ & - & $\begin{array}{c}0,90 \\
(0,88-0,92)\end{array}$ & $\begin{array}{c}0,95 \\
(0,94-0,96)\end{array}$ & - & $\begin{array}{c}0,98 \\
(0,92-1,04)\end{array}$ & $\begin{array}{c}1,02 \\
(0,94-1,10)\end{array}$ & - \\
\hline
\end{tabular}

* $\beta_{1}$ (IC95\%): inclinação da reta de regressão e intervalo de confiança a 95\%.

entre si. Ou seja, os resultados deste estudo não podem ser interpretados como se tratassem da mera concordância ou correlação entre metodologias independentes.

Enquanto estratégia de reorganização da atenção básica, o PSF preconiza a delimitação das áreas de atuação das equipes e a apropriação deste território e das necessidades de saúde da população como base para programação e execução de um conjunto de ações sanitárias de promoção, prevenção, cura e reabilitação. No processo de territorialização, podem ser citadas como etapas de importância fundamental: o cadastramento e o registro da população beneficiária do programa e melhoria dos sistemas de informação, de modo a permitir o acompanhamento das condições de saúde desta população e o sucessivo esquadrinhamento de seu território-processo.

Sendo uma estimativa da população usuária, o indicador de cobertura do PSF estima a cobertura potencial. Mas o cadastramento da população, o registro de nascidos vivos e a mensuração das famílias acompanhadas expressam a realiza- ção de ações efetivas pelas equipes para a apropriação do território de sua atuação, alicerce das atividades de promoção e prevenção da saúde, devendo, assim, ser entendidos como expressão da cobertura real do programa.

Analisar os indicadores de cadastramento e registro utilizados no estudo, não apenas como alternativa para a estimativa da população acompanhada pelas equipes de saúde da família, mas como proxy da cobertura real do programa, coloca uma dupla reflexão acerca dos achados deste estudo.

Os resultados revelam a boa adequação do indicador oficial de cobertura do PSF, a partir de 2000. Os maiores níveis de concordância observados nos grandes municípios podem ser creditados à melhor organização e maiores investimentos na gestão dos sistemas de informação nestes municípios, em especial quanto à atualização sistemática da digitação dos dados coletados pelas equipes de saúde.

Mesmo que estes achados aparentemente reforcem a manutenção de uma alternativa de estimação de cobertura que se apóia em crité- 
rios de simplicidade de cálculo, o estudo também forneceu claras evidências da melhoria da qualidade da informação sobre o cadastramento e acompanhamento da população coberta pelas equipes, disponíveis em um sistema de informação de âmbito nacional. Estes achados colocam em questão a pertinência da utilização de um indicador de cobertura obtido a partir de um parâmetro nacional único para um país de realidades tão diversas como o Brasil, tendo em vista que o percentual da população cadastrada e o percentual das famílias acompanhadas pelas equipes são indicadores com maior validade conceitual e operacional para estimar a cobertura do PSF.

Assim, os resultados apresentados neste estudo podem subsidiar discussões acerca da revisão deste indicador, que vem sendo amplamente utilizado no Brasil, nas três esferas de gestão do SUS, inclusive para definir o repasse de recursos financeiros aos municípios.

Mais do que a seleção de métodos e técnicas, questões relevantes de outra natureza devem ser consideradas. Uma vez que a cobertura de ações e serviços implica a capacidade de um aparato político-administrativo em legitimar-se mediante a suposta ou real satisfação de demandas e na confrontação de várias opções tecnológicas de organização de serviços, a mensuração da cobertura tem conotação política, embora, com freqüência, seja utilizada com enfoques meramente administrativos (como critério para o cumpri- mento de metas) ou exclusivamente técnicos. Desta forma, omite-se a idéia de que a cobertura é uma relação dinâmica entre problemas de saúde (definidos tecnicamente), necessidades de saúde (percebidas pela população) e a oferta de ações que suposta ou realmente as satisfaçam ${ }^{1}$.

No caso do PSF, uma estratégia de reorganização do nível básico de atenção de um sistema de saúde, como o SUS, que se pretende universal, equânime e integral, as reflexões acerca da definição da população-alvo, de quais necessidades de saúde devem ser atendidas na atenção básica e qual o elenco de ações e serviços de saúde que deve ser implementado conferem contornos próprios e complexos aos problemas conceituais, metodológicos e operacionais relativos às estimativas de cobertura.

A problematização de questões desta natureza, através da realização de estudos como este, traz, como principais implicações, um melhor conhecimento do processo de implantação e implementação do PSF no Brasil, em um aspecto que é crucial para a gestão: a magnitude da população beneficiária das ações e serviços de saúde. Ao mesmo tempo, ao enfatizar no campo da avaliação a importância dos estudos de cobertura uma temática que não tem sido priorizada, contribui através das reflexões levantadas para o aperfeiçoamento de estratégias de estudo para esta etapa fundamental do monitoramento e avaliação de sistemas e serviços de saúde. 


\section{Resumo}

O objetivo do estudo foi avaliar a adequação do indicador utilizado no Brasil para estimar a cobertura do Programa Saúde da Família (PSF), calculado a partir de um parâmetro nacional único de número de pessoas acompanhadas por equipe. O estudo abrangeu todos os municípios brasileiros com o PSF em funcionamento no período de 1998 a 2006. Foram utilizados os métodos de correlação de Pearson, de concordância de Lin e de regressão pelo modelo de regressão de Deming. Os resultados demonstraram que, no período inicial da expansão do PSF, o indicador de cobertura utilizado não expressava a magnitude da população beneficiária do programa, apresentando valores superiores aos referentes à população cadastrada, ao número de famílias acompanhadas e ao número de nascidos vivos registrados. Paulatinamente, em especial a partir de 2000, o indicador passou a se constituir em uma boa estimativa de cobertura do PSF, embora a evolução tenha sido diferenciada segundo o porte dos municipios, sendo mais expressiva entre os maiores municípios. O estudo enfatiza a importância da definição da cobertura das ações e serviços para orientar a implementação de programas de saúde.

Programa Saúde da Família; Cobertura de Serviços de Saúde; Avaliação do Programas e Projetos de Saúde

\section{Referências}

1. Soberon G. La extensión de cobertura de los servicios de salud. Simposio. Gac Méd Méx 1998; 124:163-89.

2. Cohen E, Franco R. A linguagem dos projetos. In: Cohen E, Franco R, organizadores. Avaliação de projetos sociais. Petrópolis: Editora Vozes; 1993. p. 85-107.

3. Rossi PH, Lipsey MW, Freeman HE. Assessing and monitoring program process. In: Rossi PH, Lipsey MW, Freeman HE, editors. Evaluation: a systematic approach. Beverly Hills: Sage Publications; 2004. p. 169-201.

4. Departamento de Atenção Básica, Secretaria de Políticas de Saúde, Ministério da Saúde. Guia prático do Programa da Saúde da Família. Brasília: Ministério da Saúde; 2001.

5. Lin LI-K. A concordance correlation coefficient to evaluate reproducibility. Biometrics 1989; 45: 255-68.

6. Bland JM, Altman DG. Statistical methods for assessing agreement between two methods of clinical measurement. Lancet 1986; 1:307-10.

7. Dallal GE. The little handbook of statistical practice. Comparing two measurement devices. http://www.graduate.tourou.edu/phd/research/ General/Reference/Resources/methods/ quantitativeanalysis/TheLittleHandbookofStatisticalPractice.htm (acessado em 10/Mar/2006).

\section{Colaboradores}

R. Aquino contribuiu na revisão da literatura, na elaboração do desenho do estudo, no processamento, análise e interpretação dos resultados e redação do artigo final. M. L. Barreto colaborou na elaboração do desenho do estudo, na análise e interpretação dos resultados e revisão do artigo final.
8. Altman DG, Bland JM. Measurement in medicine: the analysis of method comparison studies. Statistician 1983; 32:307-17.

9. Ludbrook J. Comparing methods of measurement. Clin Exp Pharmacol Physiol 1997; 24:193-203.

10. Rede Interagencial de Informações para a Saúde. Indicadores básicos de saúde no Brasil: conceitos e aplicações. Brasília: Organização Pan-Americana da Saúde; 2002.

11. Ministério da Saúde. Sistema de informações sobre mortalidade (SIM) e nascidos vivos (SINASC) para profissionais do Programa Saúde da Família. 2a Ed. Brasília: Ministério da Saúde; 2004.

12. Freire HMA, Assunção RM. Projeção populacional com estimadores Bayesianos espaciais [CD-ROM]. In: Anais do XIII Encontro da Associação Brasileira de Estudos Populacionais. Campinas: Associação Brasileira de Estudos Populacionais; 2002.

Recebido em 12/Jun/2007

Versão final reapresentada em 17/Set/2007 Aprovado em 24/Set/2007 